\title{
LA CLASE PINO-JUNIPERETEA EN LA PROVINCIA DE LEON
}

\author{
Angel PENAS MERINO, Emilio PUENTE GARCIA, \\ Luis HERRERO CEMBRANOS, \\ Carmen PEREZ MORALES \& Félix LLAMAS GARCIA
}

\begin{abstract}
RESUMEN: Se ha realizado un estudio fitosociológico tanto de los sabinares montanos, como de los enebrales rastreros subalpinos y oromediterráneos, en la provincia de León, describiéndose cuatro nuevas subasociaciones: Juniperetum sabino-thuriferae quercetosum fagineae, Genisto sanabrensis-Juniperetum nanae echinospartetosum iberici y vaccinietosum uliginosi y Vaccinio myrtilli-Juniperetum nanae jasionetosum brevisepalae.
\end{abstract}

Palabras clave: Fitosociología, Pino-Juniperetea, León, España.

ABSTRATS: In this paper a phytosociological study about montanous savin forets and, subalpine and oromediterranean creeping juniper shrubs in Leon province (NW Spain) is made. Four new subassociations are described: Juniperetum sabino-thuriferae quercetosum fagineae, Genisto sanabrensis-Juniperetum nanae echinospartetosum iberici and vaccinietosum uliginosi and Vaccinio myrtilli-Juniperetum nanae jasionetosum brevisepalae.

Key words: Phytosociology, Pino-Juniperetea, Leon, Spain.

La clase Pino-Juniperetea se corresponde conceptualmente con los bosques de coníferas poco sombríos o matorrales densos, indiferentes a la naturaleza química del sustrato y formadores de humus tangel, que representan las etapas maduras de series de vegetación climatófilas o edafoxerófilas, tanto en las altas montañas como en ciertas áreas continentales mediterráneas y eurosiberianas, donde suelen tener carácter reliquial (cf. Rivas-Martínez, 1964 y 1987; Ladero \& al., 1987).

Los bosquetes de coníferas dominados por la sabina albar (Juniperus thurifera) ocupan en la provincia de León enclaves de carácter relicto geomorfológico-climático del piso montano del Sector Ubiñense-Picoeuropeano, extendiéndose entre los ríos Luna y Cea, siendo notables los existentes en Mirantes de Luna y Crémenes, si bien existen otras manchas de importancia como las de Valmartino, Anciles y Besande.

Estos bosques debieron ocupar, en épocas pretéritas, una mayor extensión en la vertiente meridional de la Cordillera Cantábrica de la que presentan en la actualidad. Dicha extensión se ha visto disminuida por la competencia, preferentemente con fa-

Trabajo realizado con cargo al Proyecto de Investigación nº 750/86 subvencionado por la Junta de Castilla y León. 
gáceas (tales como Quercus rotundifolia, Quercus faginea o Fagus sylvatica), que en la actualidad configuran nuevas etapas climácicas o edafoxerófilas como Epipactido helleborines-Fagetum, situada siempre en exposiciones norte, o Lithodoro diffusaeQuercetum rotundifoliae, de carácter permanente y que ocupa biótopos en todo semejantes a los de los sabinares que estudiamos.

Los segundos, es decir, los matorrales densos, se corresponden con los denominados enebrales rastreros, debido a la abundante presencia, formando parte de los mismos, de Juniperus communis subsp. alpina, taxon indiferente edáfico, que conjuntamente con otros como Genista sanabrensis, Vaccinium myrtillus, Vaccinium uliginosum Cytisus oromediterraneus, Daphne laureola var. cantabrica o Arctostaphylos uva-ursi, en la provincia de León, configuran diversas comunidades vegetales, con marcado carácter biogeográfico.

Los enebrales rastreros se desarrollan en el piso subalpino de la Provincia Orocantábrica y en el oromediterráneo del Sector Orensano-Sanabriense, sobre suelos bien drenados, en crestas, cornisas, espolones rocosos y laderas con fuerte inclinación, tanto calizos como silíceos, donde la innivación es escasa, bien por el fuerte drenaje de los mismos o por el efecto del viento.

Las comunidades de la clase Pino-Juniperetea se corresponden, en nuestra provincia, con las siguientes asociaciones:

\section{Juniperetum sabino-thuriferae Rivas-Martínez, Izco \& Costa 1971}

A la presente asociación corresponden los sabinares albares del piso montano continental de la vertiente meridional de la Cordillera Cantábrica, propios del Subsector Ubiñense, en la provincia de León. Constituyen una vegetación relicta muy original que se corresponde con un bosque abierto formado por diversos estratos, en el que el superior se halla dominado por la sabina albar, Juniperus thurifera, que conjuntamente con la sabina rastrera, Juniperus sabina y el enebro enano, Juniperus communis subsp. alpina, le otorgan una peculiar combinación característica (cf. Rivas-Martínez, Izco \& Costa, 1971).

Este sabinar relicto, que presenta un abundante número de especies continentales ibéricas y meridionales eurosiberianas, ocupa los subpisos meso y altimontano de la vertiente meridional del Subsector Ubiñense (Sector Ubiñense-Picoeuropeano) de la provincia Orocantábrica, de la que es característico. Se desarrollan sobre suelos esqueléticos ricos en bases, en laderas preferentemente expuestas al Sur, donde la xericidad es acusada, a pesar de hallarse en territorios de carácter ombroclimático al menos húmedo.

La destrucción del bosque sabinero, bien por fuego o por pastoreo, conduce al desarrollo de pastizales vivaces presididos por Brachypodium pinnatum subsp. rupestre. Si la erosión se hace intensa, la roca aflorante se ve recubierta por matorrales pulviniformes dominados por Genista scorpius, Lithodora diffusa, Helianthemum croceum subsp. cantabricum, etc.

Cuando los suelos sobre los que se asienta el sabinar están más estructurados, presentando un horizonte superficial más profundo, se incorpora al mismo el quejigo, Quercus faginea, que en algunos casos se hace dominante. Para esas comunidades proponemos la subasociación quercetosum fagineae (diferencial: Quercus faginea, 
Typus: inv. 3, Tabla I), a la que habrá de referirse lo que Romero, 1983, denominó como Querco-Juniperetum thuriferae, nom. nud.

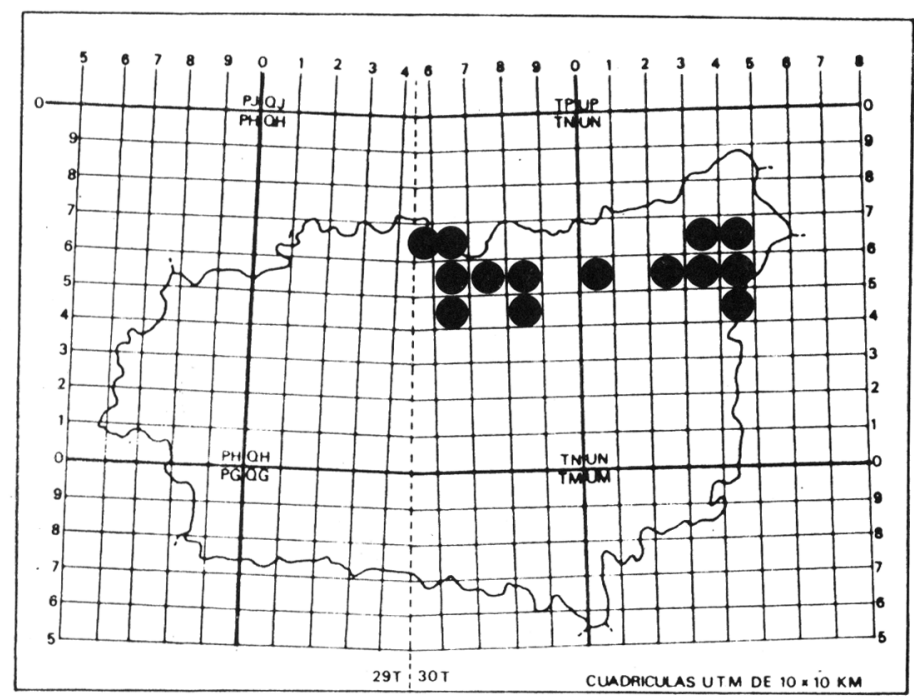

MAPA 1: Distribución en la provincia de León de las comunidades de la Juniperetum sabinothuriferae.

2. Daphno cantabricae-Arctostaphyletum uva-ursi Rivas-Martínez, Izco \& Costa 1971

En los territorios pertenecientes al piso subalpino de los Sectores UbiñensePicoeuropeano y Campurriano-Carrionés (subsector Altocarrionés), de la provincia Orocantábrica, la vegetación climácica sobre sustratos ricos en bases se corresponde con un matorral denso de porte bajo del que forman parte, entre otros táxones, Daphne laureola var. cantabrica, Arctostaphylos uva-ursi y Juniperus communis subsp. alpina, que en la actualidad y debido preferentemente a la acción del fuego, ocupan escasa extensión, siendo sus biótopos más frecuentes, cornisas y espolones rocosos, así como laderas de fuerte pendiente, donde la cobertura nival es escasa debido principalmente a los fuertes vientos existentes, a pesar de hallarse ubicados dichos matorrales en territorios con ombroclimas hiperhúmedos.

Estos enebrales rastreros, en las estaciones más continentalizadas, se ven enriquecidos con la presencia de la sabina rastrera (Juniperus sabina) que permite diferenciar la subasociación descrita por Fernández Prieto, 1983, juniperetosum sabinae, a la que corresponde el inv. $\mathrm{n}^{0} 4$ de la Tabla II.

Por otro lado, la presencia de Genista occidentalis formando parte de estas biocenosis fruticosas, nos indica el tránsito entre los subpisos subalpino inferior y altimontano que Fernández Prieto, 1983, reconoce mediante la subasociación genistetosum occidentalis (inv. $\mathrm{n}^{\circ} 5$ y 6 de la Tabla II). 


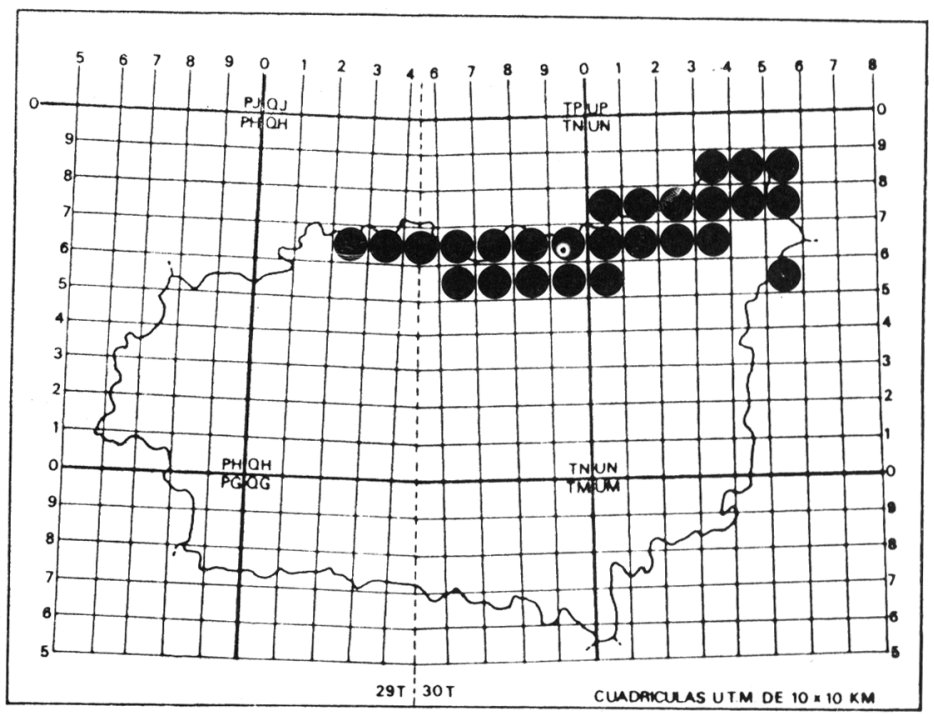

MAPA 2: Distribución en la provincia de León de las comunidades de la Daphno cantabricaeArctostaphyletum uva-ursi.

3. Junipero nanae-Vaccinietum uliginosi Rivas Goday \& Rivas Martínez ex F. Prieto 1983

Los enebrales rastreros de carácter silicícola que se desarrollan sobre suelos en los que la influencia de una materia orgánica ácida y el ombroclima hiperhúmedo, hace que tiendan a lixiviarse activamente, y que alcanzan el equilibrio en las laderas y crestas en el ranker mull o podsol férrico-húmico, están constituidos por especies fruticosas tales como Juniperus communis subsp. alpina, Vaccinium myrtillus, Vaccinium uliginosum e incluso Calluna vulgaris.

Estos matorrales densos, se desarrollan en el piso subalpino de las montañas silíceas de la Provincia Orocantábrica, y a ellos corresponden los 11 inventarios de Tabla III, si bien, los comprendidos entre el $n^{\circ} 6$ y $n^{\circ} 11$ se incluyen en la subasociación ericetosum tetralicis (cf. Fernández Prieto, 1983), que representa un particular biótopo de marcado carácter higrófilo, causado por una mayor permanencia de la nieve.

\section{Vaccinio myrtilli-Juniperetum nanae Rivas-Martínez 1964}

La fuerte continentalidad, así como una notable influencia mediterránea existente en los territorios del Subsector Laciano-Narceense, y más concretamente en los pertenecientes a la Sierra de Villabandín, límite natural entre las comarcas de Omaña y Babia, con alturas superiores a los $2.000 \mathrm{~m}$., tales como Pico Arenas $(2.100 \mathrm{~m})$, Alto de la Cañada (2.100 m), Peña Valgrán (2.030) o Pico Penouta (2.014 m), determina la presencia, sobre sustratos ácidos del piso subalpino con ombroclimas húmedo superior a hiperhúmedo, de enebrales rastreros como etapa climácica con una estructura constituida por nanofanerófitos, caméfitos arbustivos y hemicriptófitos. 


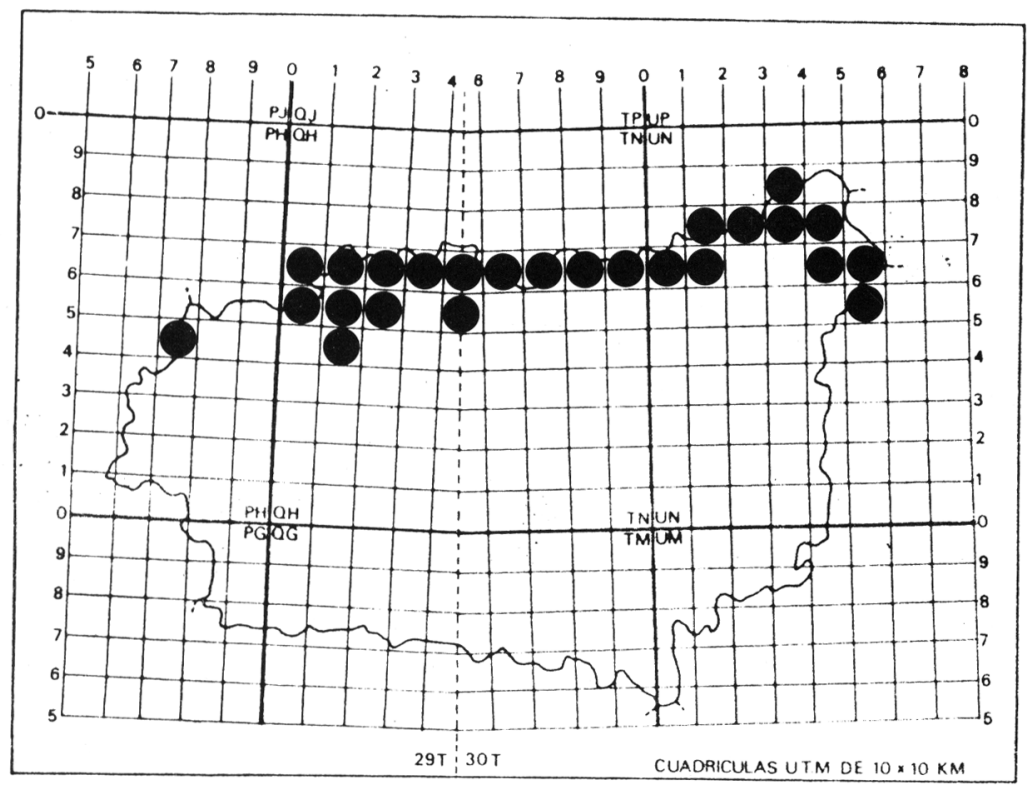

MAPA 3: Distribución en la provincia de León de las comunidades de la Junipero nanaeVaccinietum uliginosi.

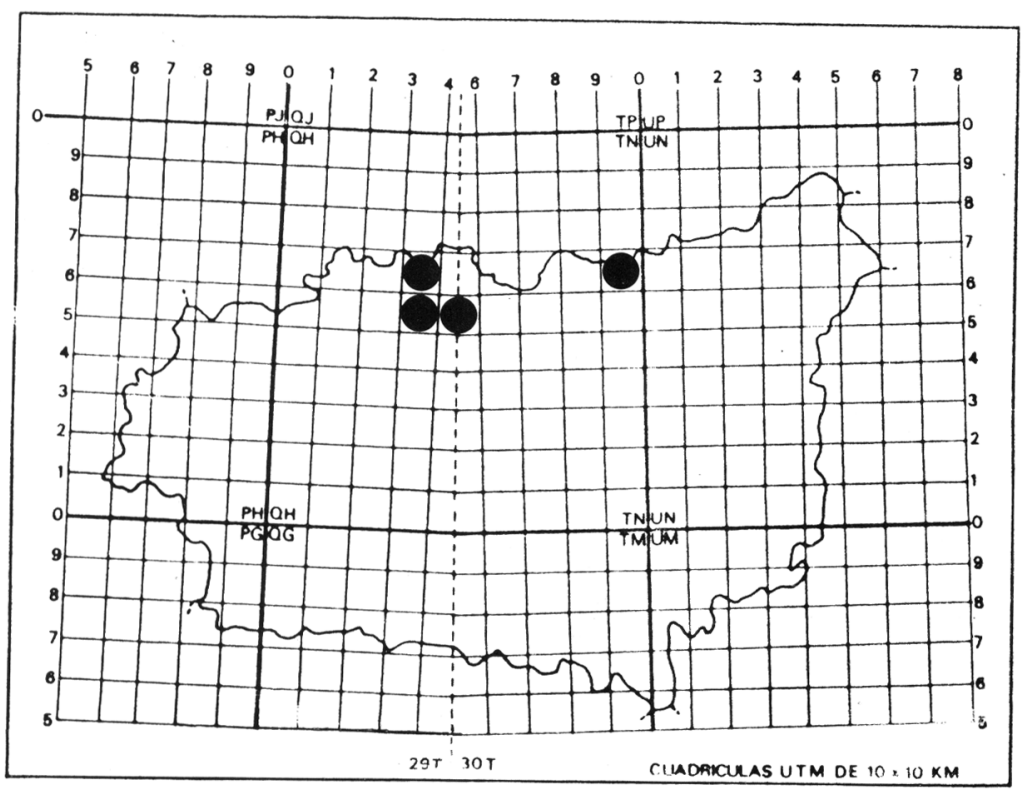

MAPA 4: Distribución en la provincia de León de las comunidades de la Vaccinio myrtilliJuniperetum nanae. 
Estas biocenosis fruticosas, presentan una combinación florística peculiar para estos territorios orocantábricos, ya que si bien se hallan presentes táxones como $J u$ niperus communis subsp. alpina, Vaccinium myrtillus, Deschampsia flexuosa subsp. iberica y Cytisus oromediterraneus, comunes en todos ellos, se hace notar la ausencia de Vaccinium uliginosum, taxon característico en estas comunidades en el resto de los territorios orocantábricos.

Dicha combinación florística, recuerda mucho, a la de los enebrales rastreros ibérico-sorianos del Vaccinio myrtilli-Juniperetum nanae (cf. Rivas-Martínez \& al., 1987 b), si bien la presencia de Jasione crispa subsp. brevisepala y Phalacrocarpum oppositifolium, táxones de marcado carácter occidental ibérico y del endemismo pirenaico-cantábrico Hypericum richeri subsp. burseri, determina una fuerte originalidad de estos matorrales climácicos frente a la asociación típica, por lo que proponemos para los mismos una nueva subasociación, jasionetosum brevisepalae (Typus: inv. $\mathrm{n}^{\mathrm{o}}$ 5, Tabla IV).

Consideramos que la abundante presencia de Vaccinium myrtillus y Calluna vulgaris en la composición de estos enebrales, táxones escasamente representados en los territorios guadarrámicos, no permite su relación con la asociación Junipero nanae-Cytisetum oromediterranei (cf. Rivas-Martínez \& al., 1987 a).

\section{Genisto sanabrensis-Juniperetum nanae F. Prieto 1983}

En el piso oromediterráneo del Sector Orensano-Sanabriense (Provincia Carpetano-Ibérico-Leonesa) representado en los Macizos de Trevinca, Teleno, Sierra Cabrera, La Guiana, etc, por encima de los 1.600 m.s.n.m., dependiendo de la exposición, latitud y mayor o menor influencia del clima atlántico, nos encontramos, sobre sustratos pobres en bases, matorrales densos presididos por el endemismo Genista sanabrensis, de los que forman parte, entre otros táxones, Juniperus communis subsp. alpina, Vaccinium myrtillus y Cytisus oromediterraneus.

Estos aulagar-enebrales representan la etapa madura en dichos territorios, cuyo ombroclima es siempre de carácter hiperhúmedo, y se desarrollan sobre litosuelos cuarcíticos o pizarrosos. Los primeros, son causa primordial para la presencia en estas formaciones de Echinospartum ibericum, taxon de marcado carácter xerófilo y cuya presencia define el tránsito entre los subpisos supramediterráneo superior y oromediterráneo inferior, siendo en este último donde se incorpora a la presente comunidad, por lo que proponemos para esta combinación florística, una nueva subasociación, echinospartetosum iberici (Typus: inv. $\mathrm{n}^{\circ} \mathbf{9}$, Tabla III).

Por otro lado, en exposiciones norte, la larga permanencia de la nieve determina una acusada hidromorfía temporal, como es el caso de la cumbre de Peña Trevinca, lo que ha permitido el refugio de Vaccinium uliginosum formando parte de este tipo de comunidades, para las cuales proponemos una nueva subasociación, vaccinietosum uiiginosi (Typus: inv. $\mathrm{n}^{\mathrm{o}} 10$, Tabla V). 


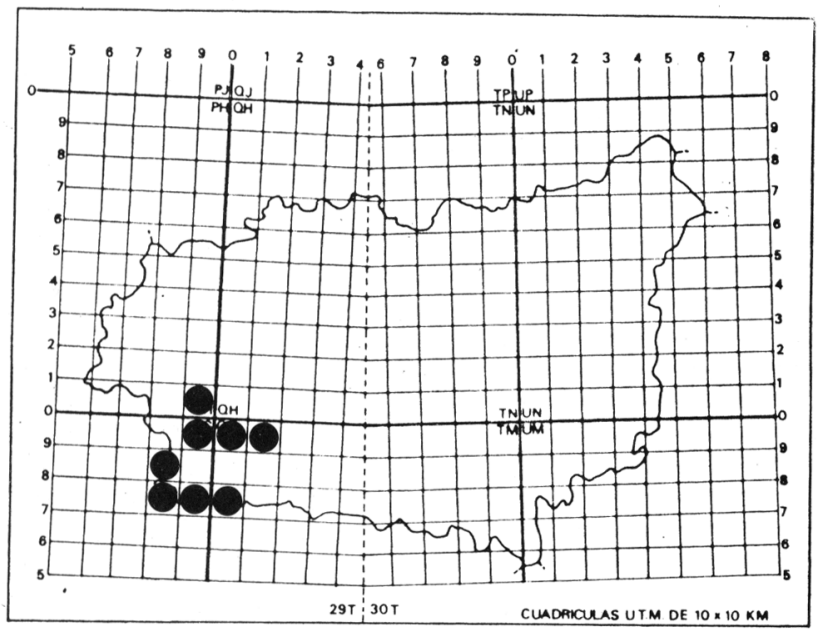

MAPA 5: Distribución en la provincia de León de las comunidades de la Genisto sanabrensisJuniperetum nanae.

\section{N D I C E S I N TA X O N O M I C O}

PINO-JUNIPERETEA Rivas-Martínez 1964

Pino-Juniperetalia Rivas-Martínez 1964

Pino-Juniperenalia ·

Cytision oromediterranei R. Tx. in R. Tx. \& Oberdorfer 1958 corr. Rivas-Martínez \& al. 1987 Cytisenion oromediterranei

Vaccinio myrtilli-Juniperetum nanae Rivas-Martínez 1964

a. jasionetosum brevisepalae subas. nova

Genisto sanabrensis-Juniperetum nanae F. Prieto 1983

a. juniperetosum nanae

b. echinospartetosum iberici subas. nova

c. vaccinietosum uliginosi subas. nova

Juniperion thuriferae Rivas-Martínez 1962

Juniperetum sabino-thuriferae Rivas-Martínez, Izco \& Costa 1971

a. juniperetosum thuriferae

b. quercetosum fagineae subas. nova

Juniperenalia nanae Rivas-Martínez, Belmonte, Cantó, F. Fernández, V. Fuente, Moreno,

Sánchez-Mata \& G. Sancho 1987

Juniperion nanae Br.-Bl. 1939

Daphno cantabricae-Arctostaphyletum uva-ursi Rivas-Martínez, Izco \& Costa 1971

a. arctostaphyletosum uva-ursi

b. juniperetosum sabinae F. Prieto 1983

c. genistetosum occidentalis F. Prieto 1983

Junipero nanae-Vaccinietum uliginosi Rivas Goday \& Rivas-Martínez ex F. Prieto 1983

a. vaccinietosum uliginosi

b. ericetosum tetralicis $\mathrm{F}$. Prieto 1983 


\section{A P E N D I C E F L O R IS T I C O}

Todos los táxones que se mencionan en el texto y en las tablas del presente trabajo están en concordancia con las propuestas de Flora Ibérica (S. Castroviejo \& al., eds. 1986) y en su defecto de Flora Europaea (T.G. Tutin \& al., eds. 1964-1980) excepto en los siguientes casos:

Agrostis tileni Nieto Feliner \& Castroviejo, Anal. Jard. Bot. Madrid 40(2):472 (1984)

Arenaria aggregata (L.) Loisel subsp. racemosa (Willk.) F.Q. var. cantabrica F.Q., Inst. Est.

Cat. Arx. Sec. Cienc. 15 (1948)

Arenaria grandiflora L. subsp. incrassata (Lange) C. Vic., Anal. Jard. Bot. Madrid 6(2):29 (1946)

Astragalus sempervirens Lam. subsp. catalaunicus (Br.-Bl.) Laínz, Candollea 24(2):254 (1969)

Avenula pratensis (L.) Dumort. subsp. iberica (St.-Yves) Romero-Zarco, Lagascalia 13(1):88 (1984)

Avenula sulcata (Gay ex Boiss,) Dumort., Bull. Soc. Bot. Belg. 7(1):128 (1868)

Berberis vulgaris L. subsp. cantabrica Rivas-Martínez, T.E. Díaz, F. Prieto, Loidi \& Penas 1984, Veg. Picos Europa: 257 (1984)

Betula pubescens Ehrh. subsp. celtiberica (Rothm. \& Vasc.) Rivas-Martínez, Trab. Dep. Bot. y F. Veg. 3:78 (1971)

Conopodium majus (Gouan) Loret subsp. ramosum (Costa) S. Silvestre, Lagascalia 2(2):151 (1972)

Cytisus oromediterraneus Rivas-Martínez, T.E. Díaz, F. Prieto, Loidi \& Penas, Veg. Picos Europa 264 (1984)

Daphne laureola L. var. cantabrica (Willk.) Willk., Suppl. Prodr. Fl. Hisp. 68 (1893)

Deschampsia flexuosa (L.) Trin subsp. iberica Rivas-Martínez, Trab. Dep. Bot. y F. Veg. 3:113 (1971)

Echinospartum ibericum Rivas-Martínez, Sánchez-Mata \& Sancho, Lazaroa 7:111 (1987)

Erica australis L. subsp. aragonensis (Wk.) P. Cout., Not. Fl. Port. (1916).

Festuca summilusitana Franco \& Rocha Afonso, Bol. Soc. Brot., 54:94 (1980).

Genista occidentalis (Rouy) Coste, Fl. Fr. 1:298 (1901)

Genista sanabrensis Valdes-Bermejo, Castroviejo \& Casaseca, Trab. Dep. Bot. Salamanca 7:5 (1978)

Genistella tridentata (L.) Samp., Herb. Port. 62 (1913)

Gentiana lutea L. subsp. aurantiaca Laínz, Inst. Est. Ast. 61-62 (1982)

Helianthemum croceum (Desf.) Pers. subsp. cantabricum Laínz, Bol. Inst. Est. Ast. ser. C. 10:20 (1964)

Hypnum cupressiforme Hedw., Sp. Musc. (1801)

Jasione crispa (Purret) Samp. subsp. brevisepala (Rothm.) Rivas-Martínez, Candollea 31:112 (1976)

Jasione laevis Lam. subsp. carpetana (Boiss. \& Reuter) Rivas-Martínez, Publ. Inst. Biol. Apl. (Barcelona) 42:122 (1967)

Juniperus communis L. subsp. alpina (Smith) Celakovsky, Prodr. Fl. Bohm. 1867

Poa alpina L. subsp. legionensis (Laínz) Rivas-Martínez, T.E. Díaz, F. Prieto, Loidi \& Penas 1984, Veg. Picos Europa 279 (1984)

Sempervivum vicentei Pau subsp. cantabricum (J.A. Huber) Fdez. Casas \& Muñoz Garmendia, Exsiccata quaedam a novis nuper distributa 1:15, n 104 (1978)

Teucrium chamaedrys L. subsp. pinnatifidum (Senn.) Rech., Bot. Arch. (Berlín) 42 (1941)

Thymus praecox Opiz subsp. britannicus (Ronn.) Holub, Preslia 45(4):359 (1973) 


\section{B I B L I O G R A F I A}

FERNANDEZ PRIETO, J.A. -1983- Aspectos geobotánicos de la Cordillera Cantábrica. Anales Jard. Bot. Madrid, 39(2):489-513.

LADERO, M., DIAZ, T.E., PENAS, A., RIVAS-MARTINEZ, S. \& VALLE, C. -1987Datos sobre la vegetación de las Cordilleras Central y Cantábrica. Itinera Geobotanica, 1:1147.

RIVAS-MARTINEZ, S. -1964- Esquema de la vegetación potencial y su correspondencia con los suelos en la España Peninsular. Anal. Inst. Bot. Cavanilles, 22:341-405.

RIVAS-MARTINEZ, S. -1987- Memoria del mapa de las series de vegetación de España. 1:400.000 I.C.O.N.A. Ministerio de Agricultura, Pesca y Alimentación. Madrid.

RIVAS-MARTINEZ, S., BELMONTE, D., CANTO, P., FERNANDEZ, F., FUENTE, V., MORENO, J.M., SANCHEZ-MATA, D. \& SANCHO, L.G. -1987a- Piornales, enebrales y pinares oromediterráneos (Pino-Cytision oromediterranei) en el Sistema Central. Lazaroa, 7:93-124.

RIVAS-MARTINEZ, S., IZCO, J. \& COSTA, M. -1971- Sobre la flora y vegetación del macizo de Peña Ubiña, Trab. Dep. Bot. y F. Veg. 3:47-123.

RIVAS-MARTINEZ, S., NAVARRO, G., MENDIOLA, A. \& TARAZONA, T. -1987b- Los enebrales rastreros oromediterráneos del Sector Ibérico-soriano. Lazaroa, 7:535-547.

ROMERO, C. -1983- Flora y vegetación de la cuenca alta del río Luna (León). I.C.O.N.A. Ministerio de Agricultura, Pesca y Alimentación. Monografía, 29:1-273. Madrid.

(Aceptado para su publicación el 15 de abril de 1989)

Dirección de los autores: Departamento de Biología Vegetal. Universidad de León. 
TABLA I

Juniperetum sabino-thuriferae Rivas-Martínez, Izco \& Costa 1971

a) juniperetosum thuriferae, b) quercetosum fagineae subas. nova

(Juniperion thuriferae, Pino-Juniperenalia, Pino-Juniperetalia, Pino-Juniperetea)

$N^{2}$ de inventario

Altitud $(1=10 \mathrm{~m}$.)

Inclinación (\%)

Exposición

Cobertura (\%)

Area $\left(\mathrm{m}^{2}\right)$

№ de especies

$\begin{array}{rrrrr}1 & 2 & 3 & 4 & 5 \\ 120 & 120 & 110 & 102 & 124 \\ 20 & 20 & 30 & 80 & 50 \\ \mathrm{~S} & \mathrm{~S} & \mathrm{~S} & \mathrm{~S} & \mathrm{~W} \\ 95 & 80 & 80 & 70 & 70 \\ 20 & 50 & 70 & 50 & 20 \\ 22 & 26 & 26 & 28 & 18 \\ & & & & \\ 2.3 & 2.3 & 3.3 & 3.4 & 2.3 \\ 2.3 & 4.5 & 2.3 & +.2 & 3.3 \\ +.2 & 1.2 & . & . & 1.2 \\ +.2 & . & 1.2 & . & \\ & & & & \\ & +.2 & +.2 & +.2 & +.2\end{array}$

Características de asociación y U.S.

Juniperus thurifera

Juniperus sabina

Festuca rubra subsp. asperifolia

Juniperus communis subsp. alpina

Diferencial de la subasociación

Quercus faginea

\section{Compañeras}

Teucrium chamaedrys subsp.pinnatifidum

\begin{tabular}{|c|c|c|c|}
\hline 1.1 & 1.2 & 1.2 & 1.1 \\
\hline 1.1 & +.2 & +.2 & +.2 \\
\hline 1.2 & 1.2 & 1.2 & . \\
\hline+.2 & 1.2 & 1.1 & \\
\hline+.2 & 2.2 & +.2 & $\therefore$ \\
\hline+.2 & +.2 & & 1.1 \\
\hline 2.3 & & 1.1 & 3.4 \\
\hline$\dot{.}$ & 1.2 & 2.2 & 1.1 \\
\hline . & 1.2 & 1.1 & 1.2 \\
\hline & 1.1 & +.2 & 2.3 \\
\hline $\begin{array}{l}1.2 \\
1.2\end{array}$ & 2.2 & . & . \\
\hline $\begin{array}{r}1.2 \\
+.2\end{array}$ & +.2 & . & . \\
\hline $\begin{array}{r}+.2 \\
+.2\end{array}$ & +.2 & . & . \\
\hline $\begin{array}{l}+.2 \\
1.1\end{array}$ & . & 1.1 & . \\
\hline 1.1 & i & & \\
\hline & $\begin{array}{l}1.1 \\
+2\end{array}$ & 1.1 & +2 \\
\hline . & $\begin{array}{r}.2 \\
+.2\end{array}$ & & +.2 \\
\hline . & +.2 & & +.2 \\
\hline . & +.2 & & +.2 \\
\hline . & . & 1.2 & 2.3 \\
\hline . & . & 1.1 & 1.1 \\
\hline . & $\cdot$. & +.2 & +.2 \\
\hline . & . & +.2 & +.2 \\
\hline . & . & $\cdot$ & 1.2 \\
\hline . & . & . & +.2 \\
\hline
\end{tabular}

Helleborus foetidus

Berberis vulgaris subsp. cantabrica

Pimpinella tragium subsp. lithophila

Origanum vulgare

Avenula pratensis subsp. iberica

Brachypodium pinnatum subsp. rupestre

Lithodora diffusa

Thymus mastichina

Helianthemum canum subsp. piloselloides

Genista scorpius

Prunus mahaleb

Sorbus aria

Daphne laureola var. cantabrica

Rhamnus alpinus

Dactylis glomerata subsp. hispanica

Rosa tomentosa

Sedum sediforme

Cerastium arvense

Achillea odorata

Genista occidentalis

Arenaria grandiflora subsp. incrassata

Pteridium aquilinum

Leucanthemum vulgare

Koeleria vallesiana

Anthyllis vulneraria

24

50 70 20 8

Además: Viburnum lantana, Rosa micrantha, Hypnum cupressiforme, Arenaria montana, Conopodium majus subsp. ramosum y Aceras anthropophorum +.2 en 1; Matthiola perennis 2.2, Prunus spinosa y Melica ciliata subsp. magnolii 1.2 y Helianthemum croceum subsp. cantabricum y Helichrysum stoechas +.2 en 2 ; Rosa nitidula y Geum sylvaticum 1.2, Clinopodium vulgare, Piptatherum paradoxum y Legousia castellana 1.1 y Centranthus lecoqii y Lactuca tenerrima +.2 en 3; Carex pilulifera 2.3, Ononis spinosa. Potentilla crantzii. Acinos alpinus y Bromus erectus 1.1 y Saponaria ocymoides, Hippocrepis comosa y Thymus mastigophorus +.2 en 4 y Asperula cynanchica. Ligusticum lucidum, Rosa canina, Arenaria aggregata subsp. racemosa var. cantabrica, Xeranthemum inapertum. Senecio minutus y Aristolochia longa +.2 en 5 .

Localidades: 1.- Barrios de Luna (30TTN64) (León). 2.- Mirantes de Luna (30TTN65) (León). 3.- Paradilla (30TTN75) (León). 4.- Crémenes (30TUN25) (León). 5.- Geras de Gordón (30TTN75) (León). 


\section{TABLA II}

Daphno cantabricae-Arctostaphyletum uva-ursi Rivas-Martínez, Izco \& Costa 1971 a) arctostaphyletosum uva-ursi, b) juniperetosum sabinae F. Prieto 1983, c) genistetosum occidentalis F. Prieto 1983

(Juniperion nanae, Juniperenalia nanae, Pino-Juniperetalia, Pino-Juniperetea)

№ de inventario

Altitud ( $1=10 \mathrm{~m}$.)

Inclinación (\%)

Exposición

Cobertura (\%)

Area $\left(\mathrm{m}^{2}\right)$

$\mathrm{N}^{\mathrm{o}}$ de especies

$\begin{array}{rrrrrr}1 & 2 & 3 & 4 & 5 & 6 \\ 160 & 165 & 155 & 134 & 154 & 191 \\ 45 & 30 & 20 & 10 & 30 & 10 \\ \mathrm{~N} & \mathrm{~N} & \mathrm{~N} & \mathrm{SW} & \mathrm{N} & \mathrm{N} \\ 60 & 90 & 100 & 100 & 100 & 100 \\ 20 & 15 & 6 & 50 & 6 & 30 \\ 10 & 7 & 10 & 7 & 10 & 8\end{array}$

Características de asociación y U.S.

Daphne laureola var. cantabrica

Juniperus communis subsp. alpina

Arctostaphylos uva-ursi

Cotoneaster integerrimus

$\begin{array}{rrrrrr}1.1 & 1.2 & +.2 & 1.2 & 1.1 & 1.2 \\ 1.2 & 3.3 & +.2 & 3.4 & 3.4 & 2.3 \\ 1.2 & 2.3 & . & 1.2 & . & 3.4 \\ . & +.2 & . & . & . & \end{array}$

Diferenciales de las subasociaciones

Juniperus sabina

Genista occidentalis

\section{Compañeras}

$\begin{array}{lllllll}\text { Helianthemum croceum subsp. cantabricum } & . & +.2 & 1.1 & \text {. } & 1.1 & +.2\end{array}$ Avenula pratensis subsp. iberica

Festuca indigesta

Hutchinsia alpina subsp. auerswaldii

Matthiola perennis

Saxifraga paniculata

Festuca hystrix

Astragalus sempervirens subsp. catalaunicus

. 1.1

2.2

1.1

1.1

$1.1+.2$
+.2

+.2 . +.2

$+.2$

$+.2$

$+.2$

$+.2$

$2.3 \quad 1.2$

Además: Poa nemoralis 1.1 y Helleborus viridis subsp. occidentalis, Lithodora diffusa, Festuca rubra, Anthyllis vulneraria, Stellaria holostea y Crepis lampsanoides +.2 en 1; Bromus erectus 1.1 en 2 ; Koeleria vallesiana +.2 en 3 ; Helianthemum canum subsp. piloselloides +.2 en 4 y Sideritis hyssopifolia +.2 en 6 .

Localidades: 1.- Pico Bodón (Valdelugueros) (30TUN05) (León). 2.- Entre Quejo y Cacabillo (29TQH26) (León). 3 y 5.- Fontún (30TTN85) (León). 4 y 6.- La Cueta (29TQH26) (León). 
TABLA III

Junipero nanae-Vaccinietum uliginosi Rivas-Goday \& Rivas-Martínez ex F. Prieto 1983 a) vaccinietosum uliginosi, b) ericetosum tetralicis F. Prieto 1983 (Juniperion nanae, Juniperenalia nanae, Pino-Juniperetalia, Pino-Juniperetea)

№ de inventario

Altitud $(1=10 \mathrm{~m}$.

Inclinación (\%)

Exposición

Cobertura (\%)

Area $\left(\mathrm{m}^{2}\right)$

$\mathrm{N}^{2}$ de especies

Características de asociación y U.S.

Juniperus communis subsp. alpina

Deschampsia flexuosa subsp. iberica
Vaccinium uliginosum

$\begin{array}{rrrrrrrrrrr}1 & 2 & 3 & 4 & 5 & 6 & 7 & 8 & 9 & 10 & 11 \\ 197 & 200 & 185 & 183 & 182 & 185 & 217 & 180 & 156 & 195 & 197 \\ 25 & 20 & 15 & 5 & 5 & 20 & 10 & 5 & . & 30 & 10 \\ \mathrm{E} & \mathrm{N} & \mathrm{N} & \mathrm{N} & \mathrm{NE} & \mathrm{N} & \mathrm{SW} & \mathrm{NE} & . & \text { NW } & \text { NW } \\ 70 & 70 & 100 & 80 & 80 & 70 & 100 & 90 & 100 & 80 & 95 \\ 20 & 20 & 25 & 10 & 10 & 70 & 100 & 10 & 10 & 10 & 20 \\ 8 & 6 & 10 & 15 & 16 & 10 & 12 & 13 & 22 & 9 & 10\end{array}$

$\begin{array}{lllllllllll}3.3 & 3.3 & 3.4 & 3.4 & 3.3 & 2.3 & 3.4 & 1.2 & +.2 & +.2\end{array}$ $\begin{array}{lllllllllll}3.3 & 2.3 & 1.2 & +.2 & 1.2 & 3.4 & 2.2 & 4.4 & 3.3 & 3.4\end{array}$ $\begin{array}{llllllll}2.2 & +.2 & 1.1 & 1.2 & +.2 & 1.1 & 1.2 & +.2\end{array}$ $\begin{array}{llllll}+.2 & +.2 & 1.2 & 1.2 & 2.3 & 2.3\end{array}$

$\begin{array}{lllllllllll}3.3 & 1.1 & 2.3 & 2.3 & 3.4 & 2.2 & 1.1 & 3.3 & 4.4 & 2.3 & 3.3\end{array}$

$\begin{array}{lllllllllll}+.2 & 1.2 & 1.2 & 1.2 & 2.3 & +.2 & 2.2 & 2.2 & 1.1 & 2.2 & 2.2\end{array}$

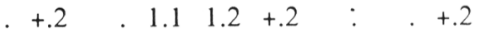

$\begin{array}{llll}1.2 & 1.2 & 1.2+.2 & 2.2\end{array}$

$1.2 . \quad . \quad+.2+.2$

+.2 . . . . +.21 .1$

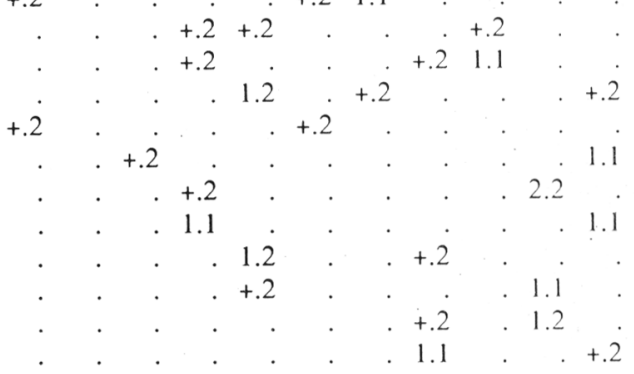

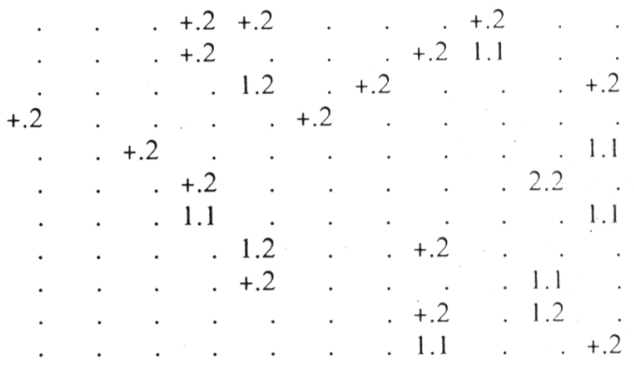

Potentilla erecta

Hypericum richeri subsp. burseri

Gentiana verna

Trifolium alpinum

Jasione crispa subsp. brevisepala

Leontodon pyrenaicus subsp.cantabricus

Galium saxatile

Plantago alpina

Poa alpina subsp. legionensis

Scilla verna

Además: Pulsatilla alpina, Phalacrocarpum oppositifolium y Allium victorialis 1.1 en 3; Carex asturica 1.2, Festuca eskia, Sedum anglicum subsp. pyrenaicum y Senecio durieui +.2 en 4; Luzula caespitosa 2.2 y Leontodon hispidus, Lotus corniculatus, Melampyrum pratense y Meum athamanticum +.2 en 5; Minuartia recurva 1.2 y Thymelaea dendrobi yum y Alchemilla saxatilis +.2 en 7 ; Juncus squarrosus +.2 en 8 ; Hieracium pilosella, Conopodium majus subsp. ramosum, Lithodora diffusa 1.1 y Anthoxanthum odoratum, Avenula sulcata, Trifolium repens, Hypochoeris radicata, Festuca iberica, Achillea millefolium, Thymus praecox subsp. britannicus, Arenaria montana y Poa nemoralis +.2 en 9; Ranunculus bulbosus subsp. castellanus 1.1 en 10 y Luzula nutans 1.1 en 11.

Localidades: 1.- Muxavén (Lumajo) (29TQH26) (León). 2 y 6.- Cueto de Arbás (Leitariegos) (29TQH06) (León). 3.- Cueto del Oso (Tejedo del Sil) (29TQH05) (León). 4 y 8.- Cueto Negro (30TTN76) (León). 5.- Catoute (29TQH14) (León). 7.- Cornón (29TQH16) (León). 9.- Puerto de Vegarada (30TTN96) (León). 10.- Salentinos (29TQH।4) (León). 11.- Peña Cuiña (29TPH74) (León). 
TABLA IV

Vaccinio myrtilli-Juniperetum nanae jasionetosum brevisepalae subas. nova (Cytisenion oromediterranei, Cytision oromediterranei, Pino-Juniperenalia, PinoJuniperetalia, Pino-Juniperetea)

$\mathrm{N}^{\circ}$ de inventario
Altitud $(1=10 \mathrm{~m}$.
Inclinación $(\%)$
Exposición
Cobertura $(\%)$
Area $\left(\mathrm{m}^{2}\right)$
$\mathrm{N}^{\circ}$ de especies

Características de asociación y U.S. Juniperus communis subsp. alpina Deschampsia flexuosa subsp. iberica Cytisus oromediterraneus

Diferenciales de la subasociación Jasione crispa subsp. brevisepala Phalacrocarpum oppositifolium Hypericum richeri subsp. burseri

\section{Compañeras}

Vaccinium myrtillus

Calluna vulgaris

Solidago virgaurea

Gentiana lutea subsp. aurantiaca

Sempervivum vicentei subsp. cantabricum

Jurinea humilis

Carex asturica

Antennaria dioica

Luzula caespitosa

Silene elegans

$\begin{array}{rrrrrr}1 & 2 & 3 & 4 & 5 & 6 \\ 184 & 201 & 199 & 200 & 197 & 208 \\ 10 & 10 & 5 & . & . & . \\ \mathrm{N} & \mathrm{SW} & \mathrm{S} & . & . & . \\ 100 & 90 & 90 & 100 & 100 & 100 \\ 200 & 50 & 100 & 150 & 100 & 100 \\ 8 & 10 & 17 & 13 & 12 & 10\end{array}$

$\begin{array}{rrrrrr}3.3 & 2.3 & 3.3 & 4.4 & 4.5 & 4.5 \\ 1.2 & 1.1 & 1.2 & 2.2 & 2.3 & 2.2 \\ 2.2 & 1.2 & 2.2 & . & 1.2 & \end{array}$

$\begin{array}{llllll}2.2 & 1.1 & 1.2 & +.2 & +.2 & 1.2\end{array}$

$\begin{array}{llllll}1.1 & 1.1 & 1.1 & 1.1 & 1.1 & 1.1\end{array}$

$\begin{array}{llllll}1.1 & +.2 & 1.2 & +.2 & 1.1 & 1.1\end{array}$

$\begin{array}{rrrrrr}3.3 & 3.4 & . & 1.1 & 1.1 & 1.2 \\ 3.3 & . & 1.2 & 2.3 & 1.2 & +.2 \\ . & 1.1 & 1.1 & 2.2 & 1.1 & +.2 \\ . & 1.1 & +.2 & . & 1.1 & . \\ . & 2.2 & +.2 & . & . & 1.2 \\ . & . & 1.1 & 1.2 & +.2 & . \\ . & . & 1.2 & 1.2 & . & . \\ . & . & 1.2 & 1.2 & . & . \\ . & . & 1.2 & . & . & +.2 \\ . & . & . & 1.1 & +.2 & .\end{array}$

Además: Eryngium durieui, Erica australis subsp. aragonensis y Genista obtusiramea +.2 en 3 y Festuca summilusitana +.2 en 4 .

Localidades: 1.- Alto de Ocedo (Sierra de Villabandín) (29TQH35) (León). 2.- Peña Valgrán (Sierra de Villabandín) (29TQH35) (León). 3 y 4.- Collado entre Peña Valgrán y Pico Arenas (29TQH35) (León). 5 y 6.- Pico Arenas (Sierra de Villabandin) (29TQH35) (León). 


\section{TABLA V}

Genisto sanabrensis-Juniperetum nanae F. Prieto 1983

a) juniperetosum nanae, b) echinospartetosum iberici subas. nova, c) vaccinietosum uliginosi subas. nova

(Cytisenion oromediterranei, Cytision oromediterranei, Pino-Juniperenalia, Pino-

Juniperetalia, Pino-Juniperetea)

№ de inventario

Altitud $(1=10 \mathrm{~m}$.

Inclinación (\%)

Exposición

Cobertura (\%)

Area $\left(\mathrm{m}^{2}\right)$

$\mathrm{N}^{\circ}$ de especies

$\begin{array}{rrrrrrrrrr}1 & 2 & 3 & 4 & 5 & 6 & 7 & 8 & 9 & 10 \\ 198 & 208 & 212 & 185 & 190 & 207 & 180 & 185 & 187 & 209 \\ 15 & 20 & 25 & 15 & 35 & 20 & 30 & 20 & 15 & 20 \\ \mathrm{~N} & \mathrm{NW} & \mathrm{S} & \mathrm{S} & \mathrm{N} & \mathrm{S} & \mathrm{N} & \mathrm{S} & \mathrm{SW} & \mathrm{NW} \\ 90 & 80 & 90 & 90 & 90 & 80 & 90 & 90 & 80 & 90 \\ 10 & 100 & 30 & 40 & 20 & 40 & 30 & 40 & 20 & 20 \\ 10 & 15 & 11 & 7 & 9 & 11 & 10 & 6 & 8 & 7\end{array}$

$\begin{array}{llllllllll}3.3 & 4.4 & 3.3 & 3.3 & 2.2 & 1.2 & 4.4 & 2.2 & 1.2 & 1.2\end{array}$

$\begin{array}{lllllllllll}2.3 & +.2 & 2.3 & 3.4 & 3.3 & 3.3 & +.2 & 3.4 & 3.4 & 3.3\end{array}$

Genista sanabrensis

Juniperus communis subsp. alpina

Cytisus oromediterraneus

\section{Diferenciales de las subasociaciones}

Echinospartum ibericum

Vaccinium uliginosum

\section{Compañeras}

Deschampsia flexuosa subsp. iberica

Agrostis tileni

Festuca summilusitana

Vaccinium myrtillus

Luzula caespitosa

Calluna vulgaris

Phalacrocarpum oppositifolium

Sedum brevifolium

Solidago virgaurea

Phyteuma hemisphaericum

Leontodon pyrenaicus subsp. cantabricus

Gentiana lutea subsp. aurantiaca

Teesdaliopsis conferta

Genistella tridentata $\begin{array}{ll}2.3 & 3.3\end{array}$

2.2

Además: Alchemilla saxatilis +.2 en 1; Silene elegans, Antennaria dioica y Jasione laevis subsp. carpetana +.2 en 2 ; Dianthus langeanus +.2 en 3; Cryptogramma crispa y Betula pubescens subsp. celtiberica +.2 en 6; Jasione crispa subsp. brevisepala 1.2 en 7 y Armeria durieui 1.2 en 9.

Localidades: 1 al 3:- Morredero (29TQG09) (León). 4 y 8.- Campa de Bercianos (29TPG88) (León). 5.- Peña Surbia (29TPG88) (León). 6, 9 y 10.- Peña Trevinca (29TPG88) (León). 7.- Sierra del Teleno (29TQG19) (León). 